\title{
Factors Associated with Physician Tolerance of Uncertainty: an Observational Study
}

\author{
Arabella Simpkin Begin, MD, MMSc ${ }^{1,2,3,4}$ (10, Michael Hidrue, PhD ${ }^{5}$, Sara Lehrhoff, MA ${ }^{5}$, \\ Marcela $G$. del Carmen, $M D, M P H^{2,5,6}$, Katrina Armstrong, MD, MSCE ${ }^{1,2}$, and \\ Jason H. Wasfy, MD, MPhil ${ }^{1,2,7}$
}

\begin{abstract}
'Department of Medicine, Massachusetts General Hospital, Boston, MA, USA; ${ }^{2}$ Harvard Medical School, Boston, MA, USA; ${ }^{3}$ Department of Pharmacology, University of Oxford, Oxford, UK; ${ }^{4}$ Division of General Internal Medicine, Massachusetts General Hospital, Boston, MA, USA; ${ }^{5}$ Massachusetts General Physicians Organization, Boston, USA; 'Division of Gynecologic Oncology, Department of Obstetrics and Gynecology and Reproductive Biology, Massachusetts General Hospital, Boston, MA, USA; ${ }^{7}$ Cardiology Division, Department of Medicine, Massachusetts General Hospital, Boston, MA, USA.
\end{abstract}

BACKGROUND: Physicians need to learn and work amidst a plethora of uncertainties, which may drive burnout. Understanding differences in tolerance of uncertainty is an important research area.

OBJECTIVE: To examine factors associated with tolerance of uncertainty, including well-being metrics such as burnout.

DESIGN: Online confidential survey.

SETTING: The Massachusetts General Physicians Organization (MGPO).

PARTICIPANTS: All 2172 clinically active faculty in the MGPO

MAIN MEASURES: We examined associations for tolerance of uncertainty with demographic information, personal and professional characteristics, and physician well-being metrics.

KEY RESULTS: Two thousand twenty (93\%) physicians responded. Multivariable analyses identified significant associations of lower tolerance of uncertainty with female gender (OR, 1.23; 95\% CI, 1.03-1.48); primary care practice (OR, 1.56; 95\% CI, 1.22-2.00); years since training (OR, 0.99; 95\% CI, 0.98-0.995); and lacking a trusted advisor (OR, 1.25; 95\% CI, 1.03-1.53). Adjusting for demographic and professional characteristics, physicians with low tolerance of uncertainty had higher likelihood of being burned-out (OR, 3.06; 95\% CI, 2.41-3.88), were less likely to be satisfied with career (OR, 0.37; 95\% CI, 0.260.52 ), and less likely to be engaged at work (RR, $0.87 ; 95 \%$ CI, 0.84-0.90).

CONCLUSION: At a time when concern about physician well-being is high, with much speculation about causes of burnout, we found a strong relationship between tolerance of uncertainty and physician well-being, across specialties. Particular attention likely needs to be paid to those with less experience, those in specialties with high rates of undifferentiated illness and uncertainty, such as primary care, and ensuring all physicians have access to a trusted advisor. These results generate the potential hypothesis that efforts focused in understanding and embracing uncertainty could be potentially effective for re-

Prior Presentations None.

Received February 4, 2021

Accepted March 29, 2021

Published online April 26, 2021 ducing burnout. This concept should be tested in prospective trials.

KEY WORDS: uncertainty; burnout; well-being; faculty development; continuing medical education.

J Gen Intern Med 37(6):1415-21

DOI: $10.1007 / \mathrm{s} 11606-021-06776-8$

(C) Society of General Internal Medicine 2021

\section{INTRODUCTION}

Although uncertainty is fundamental to medicine, the culture of medicine too often does not acknowledge uncertainty. ${ }^{1}$ The unprecedented Covid-19 pandemic has brought into clear focus our human struggle with uncertainty, with concerns raised about the toll this will have on physician well-being and burnout. Large bodies of research have demonstrated that uncertainty provokes fear, worry and anxiety, perceptions of vulnerability, and avoidance of decision-making. Stress from uncertainty is increasingly recognized as a likely driver of burnout in healthcare. ${ }^{1-4}$ Unfortunately, it is an attribute that is missing from most studies on burnout. Understanding individual differences in people's responses to-or tolerance of - uncertainty is an increasingly important focus of research. As uncertainty borders the edge of knowledge, its presence in medicine is likely to increase exponentially. Responding to the plethora of uncertainties arising in healthcare in an adaptive way is one of the most important challenges facing clinicians. ${ }^{1}$

Tolerance of uncertainty is thought to be a state determined by situational or contextual factors ${ }^{5,6}$ and therefore amenable to change through an educational and experiential process, ${ }^{7}$ though there is likely influence from inherited personality traits and prior environmental influences that predisposes individuals to specific psychological responses. Findings relating to associations between tolerance of uncertainty and sociodemographic characteristics have been inconsistent to date. Understanding and acknowledging uncertainty and acquiring proper coping strategies is regarded as a core clinical competency for medical graduates and trainees in the US, UK, Australia, and much of Europe. ${ }^{8-13}$ Further work is needed 
to ascertain how sociodemographic characteristics may be associated with varying levels of tolerance of uncertainty.

Several studies have shown an association between lower tolerance of uncertainty and increased risk or presence of provider burnout, ${ }^{2-4,14}$ but these have largely been small studies. Burnout has been described as "the index of the dislocation between what people are and what they have to do." ${ }^{15}$ It makes intuitive sense, therefore, that stress could follow from consistently having to make decisions in the face of uncertainty especially as few strategies or training opportunities exist in medical education to help physicians embrace uncertainty. Understanding factors associated with burnout is an important step to enhancing physician well-being, with downstream consequences for high-quality patient care. ${ }^{16-19}$ Physicians who are intolerant to uncertainty are reluctant to disclose their uncertainties to patients when making decisions, which can impede open, honest, and respectful communication. ${ }^{20}$ Findings on the association between tolerance of uncertainty and health services utilization have been mixed and focused on screening tests, with many showing that higher tolerance of uncertainty results in greater utilization of tests. ${ }^{21}$,

${ }^{22}$ Several studies have explored the relationship between tolerance of uncertainty and health outcomes, generally showing tolerance of uncertainty to be associated with positive outcomes. $^{23}$

To date, studies looking at physicians' tolerance of uncertainty have been mainly small-scale and single specialty with many inconsistent findings. To address this gap, we sought to examine the factors associated with physician tolerance of uncertainty, including well-being metrics such as burnout, in a large multi-speciality academic physician practice organization.

\section{METHODS}

\section{Study Design, Population, and Setting}

We conducted a cross-sectional survey of 2172 faculty in the Massachusetts General Physicians Organization (MGPO), the largest multi-specialty medical group in New England and one of the largest in the United States (US), to examine the factors associated with physician tolerance of uncertainty, particularly with respect to well-being metrics. This dataset is well positioned to answer this analytic question because to our knowledge, granular data on burnout and tolerance to uncertainty in multi-center datasets with near-complete survey responses do not exist. Data were collected in May-June 2019 as part of the biennial MGPO survey to understand physician perceptions of the functioning of the clinical enterprise within and across departments, reflecting progress made on organizational priorities, and evaluating hospital leadership. ${ }^{24-26}$ All clinically active MGPO physician members who generate more than 50 relative value units over six months were invited to participate. ${ }^{27}$ To protect confidentiality, only one analyst had access to unblinded data behind a firewall and analyses were reported without individual identifiers. All data were thus strictly anonymized so physician leaders and other administrators cannot identify respondents or link them to their survey answers. The Mass General Brigham Institutional Review Board approved this study (protocol number: 2014P002779).

\section{Survey Instrument and Variables}

Participants completed an 18-page, 30-min online survey. Survey items were developed in one of 3 ways: some were taken from previously administered faculty surveys; some domains used validated scales; and some were developed de novo using literature review and expert interviews to develop questions. Survey domains included personal and professional characteristics (e.g., gender, race, ethnicity, years of experience, specialty, and having a trusted advisor) and physician well-being metrics (e.g., overall career satisfaction, burnout, tolerance of uncertainty, work engagement, professional fulfilment, and peer support), together with other domains pertaining to compensation, administrative workload on physicians, and leadership and diversity content. ${ }^{24-26}$ All methods for the survey are in compliance with the American Association for Public Opinion Research (AAPOR) reporting guideline for survey studies. ${ }^{28}$

\section{Data Collection}

The MGPO Quality Incentive Program database provided emails for eligible faculty. A secure, web-based application, Qualtrics (Provo, Utah), was used to manage survey distribution and collect responses through email. Each participant received an individualized link to the survey. The initial email was sent on May 13, 2019, with four reminders sent to nonresponders until the survey closed on July 3, 2019. Eligibility for the incentive program is based on percentage of time physicians spend on clinical activity. Physicians earned a financial incentive for completion of the survey. ${ }^{24,25}$ Incentive amount for completing the survey ranges from $\$ 166.67$ to $\$ 833.34$ depending on the physician's amount of clinical activity. This substantial financial incentive has been associated with greater than $90 \%$ of physicians answering the survey $^{26}$ and aims to assure high response rate, minimizing potential threats to validity of survey data from differential missing data to help guide the organization's work moving forward. Physicians are asked to consent to survey participation on the cover letter inviting them to participate.

\section{Outcome Measures}

Tolerance of Uncertainty was measured using the single-item, "I find the uncertainty involved in patient care disconcerting," adapted from the 15-item Physicians' Reaction to Uncertainty Scale, originally developed by Gerrity et al. ${ }^{29}$ This single item has been shown to stratify tolerance of uncertainty in physicians, ${ }^{30-32}$ and is often used in surveys addressing multiple content areas within space constraints where use of the full 
scale is limited by its length. It ranges from 1 to 5 , with 5 signifying the greatest discomfort from uncertainty (or lowest tolerance of uncertainty). For our analysis, responses for tolerance of uncertainty were reduced to three categories: low (strongly agree or moderately agree); medium (neither agree or disagree); and high (moderately disagree and strongly disagree).

Burnout was measured using the 16-item Maslach Burnout Inventory-General Survey (MBI-GS), a validated instrument widely used to assess physician burnout. ${ }^{15}$ Per the MBI manual, respondents with scores of greater than or equal to 3.2 on the exhaustion subscale, greater than or equal to 2.6 on the cynicism subscale, or less than or equal to 3.8 on the professional efficacy subscale were defined as having high levels of burnout in that particular scale. ${ }^{15}$ For our analysis, we used a binary overall burnout measure defined as scoring high in two of the three scales of the MBI-GS. To understand potential implications of this analytic method on our results, as a sensitivity analysis, we examined a continuous measure (average score) for individual burnout scales separately.

Work Engagement was measured using the Utrecht Work Engagement Scale to measure work engagement-a positive work-related state of fulfilment that is characterized by vigor, dedication, and absorption ${ }^{33}$ (a total of 9 items on a 6-item Likert scale ranging from "never" to "daily"; range 0-54). This scale has been extensively validated in various occupational groups, including physicians. ${ }^{34}$ As recommended for this tool, we calculated mean scores for total work engagement.

Overall Career Satisfaction was measured using a 5-point Likert scale (very satisfied, satisfied, neutral, dissatisfied, or very dissatisfied) to the question "How satisfied are you with your career as a physician?" that has been used in several surveys with physicians. ${ }^{25,26}$ For our analysis, we constructed a three-level outcome: satisfied (satisfied and very satisfied); neutral; and dissatisfied (dissatisfied and very dissatisfied).

\section{Statistical Analysis}

Standard descriptive statistics were first used to characterize the sample. We used chi-square test, $t$-test, and one-way ANOVA to compare outcomes across categories, as appropriate. Multivariable regressions were used to examine the association of demographic and professional factors with outcome measures. Tolerance of uncertainty was specified as a function of gender, race, ethnicity, experience (years since training), medical specialty, and having trusted advisor. Having a trusted advisor is a five-point Likert scale question assessing whether they have a trusted advisor with whom they can discuss career goals and career satisfaction. For easier interpretation, we regrouped the responses into three categories: agree (strongly agree and agree), neutral, and disagree (strongly disagree and disagree). Physician well-being models were specified as a function of gender, race, ethnicity, experience, medical specialty, and tolerance of uncertainty. For binary outcomes, logistic regression was used; for ordinal outcomes, ordered logistic regression were used; and for continuous outcomes, generalized linear models were used. A significance level of 0.05 was used to establish statistical significance, and regression results are reported as odds ratio or rate ratio depending on the nature of outcome measure. All statistical analyses were performed using SAS version 9.4 (SAS Institute Inc., Cary, NC).

\section{RESULTS}

\section{Characteristics of the Respondents}

The overall response rate was 93\% (2020 faculty among 2172 eligible participants). Five responses were excluded due to missing data. Of the respondents, 993 (49.3\%) were male; $1186(58.9 \%)$ practiced a medical sub-specialty; 868 (43.1\%) had 10 or fewer years of experience since training; and $998(49.5 \%)$ had a trusted advisor (Table 1).

\section{Tolerance of Uncertainty and Sociodemographic Characteristics}

In bivariate analyses, we found significant difference in the distribution of tolerance of uncertainty by gender $\left(x^{2}=18.0\right.$, $p=0.001)$, experience $\left(x^{2}=16.1, p=0.013\right)$, and specialty $\left(x^{2}=17.8, p=0.007\right)$. Tolerance of uncertainty was not associated with whether the physician had a trusted advisor $\left(x^{2}=7.1\right.$, $p=0.132$ ) (Table 1).

In multivariable analyses, lower tolerance of uncertainty was associated with female gender (odds ratio [OR], 1.23; 95\% CI, 1.03-1.48); primary care practice (PCP) (OR, 1.56; 95\% CI, 1.22-2.00); and lack of a trusted advisor (OR, 1.25; $95 \% \mathrm{CI}, 1.03-1.53)$. More experience is associated with lower odds of low tolerance of uncertainty (OR, 0.99 ; $95 \%$ CI, 0.98 0.995) (Table 2).

\section{Tolerance of Uncertainty and Physician Well- being}

In bivariate analyses, physicians with low tolerance of uncertainty were more likely to be burned-out than those with high tolerance of uncertainty $(49.4 \%$ vs $23.5 \%$; $p<0.0001)$, less likely to be engaged at work (average total engagement score of 4.1 vs $4.7 ; p<0.0001$ ), and less likely to be satisfied with their career $(82.2 \%$ vs $92.7 \%$; $p<0.001)$. Similarly, physicians with low tolerance of uncertainty were more likely to have higher rates of exhaustion ( 3.5 vs $2.5 ; p<0.001)$; cynicism ( 2.5 vs $1.7 ; p<0.0001)$; and reduced personal efficacy (1.6 vs 1.1 ; $p<0.001)$.

In multivariable model, adjusting for demographic and professional characteristics, physicians with low levels of tolerance of uncertainty were more likely to be burned-out (OR, 3.06; 95\% CI, 2.41-3.88), less likely to be satisfied with their career (OR, $0.37 ; 95 \% \mathrm{CI}, 0.26-0.52$ ), and less likely to be engaged at work (RR, $0.87 ; 95 \% \mathrm{CI}, 0.84-0.90)$ than 
Table 1 Cohort characteristics and their association with tolerance of uncertainty

\begin{tabular}{|c|c|c|c|c|c|}
\hline \multirow[t]{2}{*}{ Sample characteristics } & \multirow[t]{2}{*}{ Sample distribution, $N(\%)^{*}$} & \multicolumn{4}{|c|}{$\begin{array}{l}\text { Percentage by level of tolerance of } \\
\text { uncertainty } \dagger\end{array}$} \\
\hline & & High & Medium & Low & $p$-value \\
\hline Gender & & & & & $18.0(0.001)$ \\
\hline Female & $905(44.9)$ & 48.0 & 28.1 & 23.9 & \\
\hline Male & $993(49.3)$ & 56.4 & 23.0 & 20.6 & \\
\hline Prefer not to say & $117(5.81)$ & 43.6 & 33.3 & 23.1 & \\
\hline Experience (years since training) & & & & & $16.1(0.013)$ \\
\hline$\leq 10$ & $868(43.1)$ & 47.8 & 28.6 & 23.6 & \\
\hline $11-20$ & $563(27.9)$ & 52.6 & 26.1 & 21.3 & \\
\hline $21-30$ & 357 (17.7) & 57.1 & 19.9 & 23.0 & \\
\hline$>30$ & $227(11.3)$ & 57.2 & 24.2 & 18.5 & \\
\hline Specialty & & & & & $17.8(0.007)$ \\
\hline Emergency medicine, radiology, anesthesia, and pathology & $346(17.2)$ & 48.8 & 30.9 & 20.2 & \\
\hline Medical subspecialties & 1186 (58.9) & 54.1 & 24.4 & 21.5 & \\
\hline Primary care & $294(14.6)$ & 44.2 & 26.5 & 29.3 & \\
\hline Surgical specialties & $189(9.4)$ & 55.6 & 24.3 & 20.1 & \\
\hline Having a trusted advisor & & & & & $7.1(0.132)$ \\
\hline Disagree & $617(30.6)$ & 50.1 & 24.5 & 25.4 & \\
\hline Neutral & $400(19.9)$ & 50.8 & 29.0 & 20.2 & \\
\hline Agree & $998(49.5)$ & 53.4 & 25.5 & 21.1 & \\
\hline
\end{tabular}

*These percentages represent distribution of respondents within each characteristic question

tThese percentages represent distribution of tolerance of uncertainty within a given category of a characteristic (row percentages)

physicians with high tolerance of uncertainty (Table 3). Compared to physicians with higher levels of tolerance of uncertainty, physicians with low levels of tolerance have higher rates of exhaustion (RR, 1.37; 95\% CI, 1.28-1.47), higher rates of cynicism (RR, 1.46; 95\% CI, 1.34-1.60), and higher rates of personal inefficacy (RR, 1.44; 95\% CI, 1.32-1.57) (Table 3).

\section{DISCUSSION}

This study adds new knowledge by comprehensively examining the factors associated with physician tolerance of uncertainty in a large sample of multi-specialty physicians, with a range of experience. Given this research question requires detailed and complete information about burnout, this research question is likely unanswerable from large national datasets. As such, the work here provides an ideal balance between granular survey data with minimal missing data and a large, diverse physician population to support external validity of the results. At a time when concern about faculty well-being is high, with much speculation about causes of burnout, ${ }^{35}$ we found a strong relationship between tolerance of uncertainty and physician well-being, regardless of how physician wellbeing is measured. We have not distinguished between different sources of uncertainty as uncertainty in healthcare pertains to numerous unknowns which likely co-exist and overlap. An intolerance of uncertainty has been shown to be a characteristic involved in excessive worry. ${ }^{36-38}$ The psychological distress which is associated with an intolerance of uncertainty has consequences not only for the physical and mental well-being of physicians but also has a detrimental impact on their ability to perform well academically. ${ }^{39}$ As we think about the urgent call to alleviate burnout, efforts focused in understanding and embracing uncertainty seem vital. Our study showed that female gender, PCP specialty, lack of experience, and lack of trusted advisor were associated with lower tolerance of uncertainty.

This study builds on earlier research evaluating tolerance of uncertainty and burnout in healthcare providers, with several studies showing an association between lower tolerance of uncertainty and increased risk or presence of provider burnout $^{2-4,14}$ and work-related stress. ${ }^{40-43}$ This association has been shown to be present early in training, with a study of medical students showing that intolerance of uncertainty can cause significant anxiety, frustration, self-doubt, disillusionment, feelings of inadequacy (not being "good enough"), and insecurity regarding professional skill level. ${ }^{44}$ Understanding and acknowledging uncertainty and acquiring proper coping strategies is now regarded as one of the core clinical competencies for medical graduates and trainees in the UK, US, Australia, and much of Europe, ${ }^{8-13,45}$ but there is still much about tolerance of uncertainty that is not understood. Previous

Table 2 Ordered Logistic Regression Model Predicting Association of Physicians Covariates and Tolerance of Uncertainty*

\begin{tabular}{|c|c|c|}
\hline \multirow[t]{2}{*}{ Covariates } & \multicolumn{2}{|c|}{ Odds ratio } \\
\hline & Estimate & {$[95 \% \mathrm{CI}]$} \\
\hline \multicolumn{3}{|c|}{ Gender $(\mathrm{ref}=\mathrm{male})$} \\
\hline Female & 1.23 & {$[1.03-1.48]$} \\
\hline Prefer not to say & 1.49 & {$[0.94-2.36]$} \\
\hline Experience (in years) & 0.99 & {$[0.98-0.995]$} \\
\hline \multicolumn{3}{|c|}{ Snecialty (ref=medical subsnecialties) } \\
\hline ERAPs $\dagger$ & 1.13 & {$[0.89-1.42]$} \\
\hline Primary care & 1.56 & {$[1.22-2.00]$} \\
\hline Surgical specialties & 1.03 & {$[0.76-1.39]$} \\
\hline \multicolumn{3}{|c|}{ Have a trusted advisor (ref=agree) } \\
\hline Neutral & 1.10 & {$[0.88-1.38]$} \\
\hline Disagree & 1.25 & {$[1.03-1.53$} \\
\hline
\end{tabular}

*Besides the factors in the table, we also controlled for race and ethnicity

†Emergency medicine, radiology, anesthesia, and pathology 
Table 3 Multivariable Regression Results Predicting Association of Tolerance of Uncertainty with Physician Well-being Measures*

\begin{tabular}{|c|c|c|c|c|}
\hline \multirow[t]{3}{*}{$\begin{array}{l}\text { Physician well-being } \\
\text { model }\end{array}$} & \multicolumn{4}{|c|}{$\begin{array}{l}\text { Association with tolerance } \\
\text { (reference=high) } \dagger\end{array}$} \\
\hline & \multicolumn{2}{|l|}{ Medium } & \multicolumn{2}{|l|}{ Low } \\
\hline & Estimate & $\begin{array}{l}95 \% \\
\text { CI }\end{array}$ & Estimate & $\begin{array}{l}95 \% \\
\text { CI }\end{array}$ \\
\hline Burnout rate & 1.90 & $\begin{array}{l}1.50- \\
2.40\end{array}$ & 3.06 & $\begin{array}{l}2.41- \\
3.88\end{array}$ \\
\hline Career satisfaction & 0.41 & $\begin{array}{l}0.29 \\
0.57\end{array}$ & 0.37 & $\begin{array}{l}0.26- \\
0.52\end{array}$ \\
\hline $\begin{array}{l}\text { Total work } \\
\text { engagement } \\
\text { Burnout subscales } \\
\text { (in rate ratio) }\end{array}$ & 0.91 & $\begin{array}{l}0.88- \\
0.94\end{array}$ & 0.87 & $\begin{array}{l}0.84 \\
0.90\end{array}$ \\
\hline Exhaustion & 1.23 & $\begin{array}{l}1.15- \\
1.32\end{array}$ & 1.37 & $\begin{array}{l}1.28- \\
1.47\end{array}$ \\
\hline Cynicism & 1.27 & $\begin{array}{l}1.17- \\
1.39\end{array}$ & 1.46 & $\begin{array}{l}1.34- \\
1.60\end{array}$ \\
\hline $\begin{array}{l}\text { Reduced personal } \\
\text { efficacy }\end{array}$ & 1.30 & $\begin{array}{l}1.19- \\
1.41\end{array}$ & 1.44 & $\begin{array}{l}1.32- \\
1.57\end{array}$ \\
\hline
\end{tabular}

*Burnout is specified as binary outcome and modelled using logistic regression. Career satisfaction is specified as 3-level ordinal outcome and modelled using ordered logistic regression. Results for these models are reported as odds ratio. Total work engagement and individual burnout scales are specified as continuous measures and modelled using generalized linear model with log link and gamma distribution. Results for these models are reported as rate ratio

fIn addition to tolerance to uncertainty, all models controlled for differences in gender, race, ethnicity, years of experience, and specialty. To reduce space, we present here only the results associated with low tolerance of uncertainty. Full results are available from authors

studies have been inconsistent in reporting higher levels of tolerance of uncertainty in men or women, with some reporting higher levels in women, ${ }^{40}$ some reporting higher levels in men, ${ }^{46}$ and others remaining inconclusive. ${ }^{47}$ There has also been previous inconsistency with tolerance of uncertainty and age, with some reporting increased tolerance with older age, ${ }^{48-50}$ some demonstrating tolerance is higher with younger age, ${ }^{51}$ and some showing no association. ${ }^{52}$ In this large multispecialty study, we found significantly lower levels of tolerance of uncertainty in females, PCP specialty, those with less experience, and those who lacked a trusted advisor. It is important to recognize that talking openly about uncertainty in the clinical environment helps normalize the experience of uncertainty, especially for those colleagues with less experience. ${ }^{53}$

Our findings generate the hypothesis that reducing tolerance of uncertainty might improve physician well-being. As healthcare organizations look to improve tolerance of uncertainty among their physicians, it may be helpful to focus on potentially modifiable factors associated with lower tolerance of uncertainty. Particular attention likely needs to be paid to those with less experience, and perhaps those in specialties with high rates of undifferentiated illness and uncertainty, such as primary care. Having a trusted advisor appears to be associated with higher levels of tolerance of uncertainty, which may reflect an ability for individuals to talk openly and express anxieties and concerns with a colleague in a safe space. ${ }^{53}$
Ensuring physicians have access to a trusted advisor is an easy-to-implement strategy that could pay dividends.

A provider's ability to deal with uncertainty at a cognitive, emotional, and ethical level has been shown to influence the diagnostic process with potential for diagnostic error and impact on patient outcomes. ${ }^{54}$ When confronted with a clinical situation whose consequences are not easy to predict - and this concerns most medical decision-making - high uncertainty intolerant physicians may feel particularly worried and anxious about the implications of their decisions, thus resulting in a feeling of being "stuck" in the uncertainty and unable to move forward. ${ }^{39}$ It is estimated that $17 \%$ of excessive costs in medical care result from physicians' anxiety related to how they manage uncertainty, ${ }^{55}$ with increased test-ordering tendencies ${ }^{56,}{ }^{57}$ failure to comply with evidence-based guidelines, ${ }^{58}$ and fear of malpractice litigation and defensive practice. ${ }^{59}$ These extraneous interventions not only increase healthcare costs but also place patients at risk for experiencing adverse events from unnecessary tests and treatments, ${ }^{60,} 61$ causing unnecessary concern and distress to patients. In addition, suppression of uncertainty and lack of consideration of alternative diagnoses can result in premature closure - the tendency to stop considering other possibilities after reaching an initial diagnosis - the single most common phenomenon in misdiagnosis. ${ }^{62}$ There is growing recognition of the importance of diagnostic error with regard to patient safety. Helping physicians embrace uncertainty may be an important strategy as we look to minimize diagnostic error and enhance patient safety and high-quality care.

This study has several limitations. First, our results are subject to the inherent reporting biases that often occur in survey studies. To minimize any social desirability biases, respondents were informed about extensive efforts to collect and analyze data in a de-identified and confidential manner. In addition, survey participants were unaware of the specific hypothesis of this study, and we have no information to suggest that they would have chosen to participate or not on the basis of their tolerance of uncertainty or degree of burnout. Our response rate of $93 \%$ is a robust response across a large multi-specialty group which increases the accuracy of the data and minimizes any selection bias. Second, our results may not be generalizable beyond the academic faculty practice of physicians at the MGPO. However, we are reassured that the external validity of these results is strong, since this is the largest physician organization in New England and includes physicians from a diverse range of practice settings and specialties. Nevertheless, further studies are needed to confirm our findings in other hospital and academic settings. Finally, due to the observational design of our study, we are careful to only test for associations and do not draw conclusions about causality from our findings alone.

Identifying and effectively managing physician burnout has proven to be extremely difficult, with high rates persisting in numerous studies over time, despite increased attention to this issue. This study generates the hypothesis that efforts to 
improve management of uncertainty may be useful for addressing burnout among physicians, across specialties, and gives insight into the demographic characteristics of those who may be at highest risk of stress from uncertainty.

Corresponding Author: Arabella Simpkin Begin, MD, MMSc; Division of General Internal Medicine, Massachusetts General Hospital, Boston, MA, USA (e-mail: asimpkin@mgh.harvard.edu).

Funding This study was funded in part by an award from the American Heart Association (18 CDA 34110215 awarded to Dr. Wasfy); the Massachusetts General Physicians Organization; and the Center for Educational Innovation and Scholarship at Massachusetts General Hospital. The funding sources had no role in the design of the study, the analysis and interpretation of the data, and the decision to approve publication of the finished manuscript. However, some of the authors hold leadership positions in the organization.

\section{Declarations:}

Ethics Approval: The Institutional Review Board of the organizing hospital network (Mass General Brigham, Protocol number: 2014P002779) approved the study. The Partners Human Research Committee determined the project met the criteria for Institutional Review Board exemption because the research was limited to the use of survey data, was not subject to FDA regulations, and there was not more than minimal risk to study subjects. Completion of the survey was considered implied consent of participation. All data used were strictly anonymized; only a research coordinator had access to the file linking responses to identifiers. All authors had full access to all the data (including statistical reports and tables) in the study and can take responsibility for the integrity of the data and the accuracy of the data analysis.

Conflict of Interest: The authors report no declarations of interest.

IRB Approval: The Mass General Brigham Institutional Review Board approved this study (protocol number: 2014P002779).

\section{REFERENCES}

1. Simpkin, A.L. and R.M. Schwartzstein, Tolerating Uncertainty - The Next Medical Revolution? N Engl J Med, 2016. 375(18): p. 1713-1715.

2. Simpkin, A.L., et al., Stress From Uncertainty and Resilience among Depressed and Burned Out Residents: a Cross-Sectional Study. Acad Pediatr, 2018. 18(6): p. 698-704.

3. Cooke, G.P., J.A. Doust, and M.C. Steele, A survey of resilience, burnout, and tolerance of uncertainty in Australian general practice registrars. BMC Med Educ, 2013. 13: p. 2.

4. Kuhn, G., R. Goldberg, and S. Compton, Tolerance for uncertainty, burnout, and satisfaction with the career of emergency medicine. Ann Emerg Med, 2009. 54(1): p. 106-113 e6.

5. Herman, J., et al., The tolerance for ambiguity scale: towards a more refined measure for international management research. Int J Intercult Relat, 2010. 34: p. 58-65.

6. Durrheim, K. and D. Foster, Tolerance of ambiguity as a content specific construct. Personality and Individual Differences, 1997. 22: p. 741-750.

7. Zwack, J. and J. Schweitzer, If every fifth physician is affected by burnout, what about the other four? Resilience strategies of experienced physicians. Acad Med, 2013. 88(3): p. 382-9.

8. General Medical Council: Outcomes for Graduates. 2018; Available from: https://www.gmc-uk.org/-/media/documents/dc11326-outcomes-forgraduates-2018_pdf-75040796.pdf.

9. Harden, R.M., et al., AMEE Guide No. 14: Outcome-based education: Part 5-From competency to meta-competency: a model for the specification of learning outcomes. Med Teach, 1999. 21(6): p. 546-52.

10. Simpson, J.G., et al., The Scottish doctor-learning outcomes for the medical undergraduate in Scotland: a foundation for competent and reflective practitioners. Med Teach, 2002. 24(2): p. 136-43.

11. Englander, R., et al., Toward a common taxonomy of competency domains for the health professions and competencies for physicians. Acad Med, 2013. 88(8): p. 1088-94.

12. Cumming, A.D. and M. Ross. Learning outcomes/competencies for undergraduate medical education in Europe: Tuning Project (Medicine). 2018; Available from: http://www.unideusto.org/tuningeu/images/ stories/Summary_of_outcomes_TN/Learning_Outcomes_Competences_ for_Undergraduate_Medical_Education_in_Europe.pdf.

13. Expectations for level of achievement of the graduate capabilities in each phase of the curriculum. 2011: Sydney, Australia: The University of New South Wales.

14. Kimo Takayesu, J., et al., Factors associated with burnout during emergency medicine residency. Acad Emerg Med, 2014. 21(9): p. 1031-5.

15. Maslach, C., S.E. Jackson, and M.P. Leiter, Maslach Burnout Inventory Manual. 3rd ed ed. 1996, Menlo Park, CA: Mind Garden Inc.

16. Fahrenkopf, A.M., et al., Rates of medication errors among depressed and burnt out residents: prospective cohort study. BMJ, 2008. 336(7642): p. $488-91$.

17. West, C.P., et al., Association of resident fatigue and distress with perceived medical errors. JAMA, 2009. 302(12): p. 1294-300.

18. Dyrbye, L.N. and T.D. Shanafelt, Physician burnout: a potential threat to successful health care reform. JAMA, 2011. 305(19): p. 2009-10.

19. Dewa, C.S., et al., How does burnout affect physician productivity? A systematic literature review. BMC Health Serv Res, 2014. 14: p. 325.

20. Katz, J., Why doctors don't disclose uncertainty. Hastings Cent Rep, 1984. 14(1): p. 35-44.

21. Han, P.K., et al., Individual differences in aversion to ambiguity regarding medical tests and treatments: association with cancer screening cognitions. Cancer Epidemiol Biomarkers Prev, 2014. 23(12): p. 2916-23.

22. Tambor, E.S., et al., Offering cystic fibrosis carrier screening to an HMO population: factors associated with utilization. Am J Hum Genet, 1994. 55(4): p. 626-37.

23. Strout, T.D., et al., Tolerance of uncertainty: A systematic review of health and healthcare-related outcomes. Patient Educ Couns, 2018. 101(9): p. 1518-1537.

24. Meyer, G.S., D.F. Torchiana, and D.G. Colton, The use of modest incentives to boost adoption of safety practices and systems. Advances in patient safety: new directions and alternative approaches, ed. K. Henriksen, et al. 2008, Rockville, MD: Agency for healthcare research and quality.

25. Rao, S., et al., The impact of administrative buden on academic physicians: results of a hospital-wide physician survey. Acad Med, 2017. 92(2): p. 237-243.

26. Del Carmen, M.G., et al., Trends and Factors Associated With Physician Burnout at a Multispecialty Academic Faculty Practice Organization. JAMA Netw Open, 2019. 2(3): p. e190554.

27. Torchiana, D.F., et al., Massachusetts General Physicians Organization's quality incentive program produces encouraging results. Health Aff (Millwood), 2013. 32(10): p. 1748-56.

28. American Association for Public Opinion Research. Standard definitions. Available from: https://www.aapor.org/Publications-Media/AAPORJournals/Standard-Definitions.aspx.

29. Gerrity, M.S., R.F. DeVellis, and J.A. Earp, Physicians' reactions to uncertainty in patient care. A new measure and new insights. Med Care, 1990. 28(8): p. 724-36.

30. Tilburt, J.C., et al., Views of US physicians about controlling health care costs. JAMA, 2013. 310(4): p. 380-8.

31. Colla, C.H., et al., Physician perceptions of Choosing Wisely and drivers of overuse. Am J Manag Care, 2016. 22(5): p. 337-43.

32. Ganguli, I., et al., Cascades of Care After Incidental Findings in a US National Survey of Physicians. JAMA Netw Open, 2019. 2(10): p. e1913325.

33. Schaufeli, W.B. and A.B. Bakker, The Measurement of Work Engagement with a Short Questionnaire: A Cross-National Study. Educational and Psychological Measurement, 2006. 66(4): p. 701-716.

34. Scheepers, R.A., et al., In the eyes of residents good supervisors need to be more than engaged physicians: the relevance of teacher work engagement in residency training. Adv Health Sci Educ Theory Pract, 2015. 20(2): p. 441-55.

35. Schwenk, T.L. and K.J. Gold, Physician burnout-A serious symptom, but of what? JAMA, 2018. 320(11): p. 1109-1110.

36. Ladouceur, R., F. Talbot, and M.J. Dugas, Behavioral expressions of intolerance of uncertainty in worry. Experimental findings. Behav Modif, 1997. 21(3): p. 355-71. 
37. Dugas, M.J., N. Laugesen, and W.M. Bukowski, Intolerance of uncertainty, fear of anxiety, and adolescent worry. J Abnorm Child Psychol, 2012. 40(6): p. 863-70.

38. Buhr, K. and M.J. Dugas, The role of fear of anxiety and intolerance of uncertainty in worry: an experimental manipulation. Behav Res Ther, 2009. 47(3): p. 215-23.

39. Lally, J. and P. Cantillon, Uncertainty and ambiguity and their association with psychological distress in medical students. Acad Psychiatry, 2014. 38(3): p. 339-44.

40. Bovier, P.A. and T.V. Perneger, Stress from uncertainty from graduation to retirement-a population-based study of Swiss physicians. J Gen Intern Med, 2007. 22(5): p. 632-8.

41. van der Weijden, T., et al., Understanding laboratory testing in diagnostic uncertainty: a qualitative study in general practice. Br J Gen Pract, 2002 52(485): p. 974-80.

42. Griffiths, F., E. Green, and M. Tsouroufli, The nature of medical evidence and its inherent uncertainty for the clinical consultation: qualita tive study. BMJ, 2005. 330(7490): p. 511.

43. Iannello, P., et al., Ambiguity and uncertainty tolerance, need for cognition, and their association with stress. A study among Italian practicing physicians. Med Educ Online, 2017. 22(1): p. 1270009.

44. Nevalainen, M.K., T. Mantyranta, and K.H. Pitkala, Facing uncertainty as a medical student-a qualitative study of their reflective learning diaries and writings on specific themes during the first clinical year. Patient Educ Couns, 2010. 78(2): p. 218-23.

45. (ACGME), A.C.f.G.M.E. ACGME Common Program Requirements, 2018 [cited 2018 October 13]; Available from: https://www.acgme.org/Portals/ 0/PFAssets/ProgramRequirements/CPRFellowship2019.pdf.

46. Caulfield, M., et al., Ambiguity tolerance of students matriculating to U.S. medical schools. Acad Med, 2014. 89(11): p. 1526-32.

47. Geller, G., et al., Measuring physicians' tolerance for ambiguity and its relationship to their reported practices regarding genetic testing. Med Care, 1993. 31(11): p. 989-1001.

48. DeForge, B.R. and J. Sobal, Intolerance of ambiguity in students entering medical school. Soc Sci Med, 1989. 28(8): p. 869-74.

49. Allen, A.S., et al., Clinician personality and the evaluation of higher-risk patient symptoms. J Patient Saf, 2011. 7(3): p. 122-6.
50. Callahan, P., et al., Relationship between tolerance/intolerance of ambiguity and perceived environmental uncertainty in hospitals. J Psychosoc Nurs Ment Health Serv, 1997. 35(11): p. 39-44.

51. Geller, G., R.R. Faden, and D.M. Levine, Tolerance for ambiguity among medical students: implications for their selection, training and practice. Soc Sci Med, 1990. 31(5): p. 619-24.

52. Raglan, G.B., et al., Need to know: the need for cognitive closure impacts the clinical practice of obstetrician/gynecologists. BMC Med Inform Decis Mak, 2014. 14: p. 122.

53. Gheihman, G., M. Johnson, and A.L. Simpkin, Twelve tips for thriving in the face of clinical uncertainty. Med Teach, 2019: p. 1-7.

54. Evans, L. and D.R. Trotter, Epistemology and uncertainty in primary care: an exploratory study. Fam Med, 2009. 41(5): p. 319-26.

55. Allison, J.J., et al., The association of physician attitudes about uncertainty and risk taking with resource use in a Medicare HMO. Med Decis Making, 1998. 18(3): p. 320-9.

56. Pines, J.M., et al., The effect of physician risk tolerance and the presence of an observation unit on decision making for ED patients with chest pain. Am J Emerg Med, 2010. 28(7): p. 771-9.

57. Pines, J.M., et al., The association between physician risk tolerance and imaging use in abdominal pain. Am J Emerg Med, 2009. 27(5): p. 552-7.

58. Ghosh, A.K., On the challenges of using evidence-based information: the role of clinical uncertainty. J Lab Clin Med, 2004. 144(2): p. 60-4

59. Benbassat, J., D. Pilpel, and R. Schor, Physicians' attitudes toward litigation and defensive practice: development of a scale. Behav Med, 2001. 27(2): p. 52-60.

60. Politi, M.C. and F. Legare, Physicians' reactions to uncertainty in the context of shared decision making. Patient Educ Couns, 2010. 80(2): p. 155-7.

61. Carney, P.A., et al., Reactions to uncertainty and the accuracy of diagnostic mammography. J Gen Intern Med, 2007. 22(2): p. 234-41.

62. Graber, M.L., N. Franklin, and R. Gordon, Diagnostic error in internal medicine. Arch Intern Med, 2005. 165(13): p. 1493-9.

Publisher's Note: Springer Nature remains neutral with regard to jurisdictional claims in published maps and institutional affiliations. 